\title{
Theoretical and experimental study on a finite size vector sensor array with a cylindrically symmetric carrier
}

\author{
Junyuan Guo ${ }^{1,2,3}$, Shi-e Yang ${ }^{1,2,3}$, Hongjuan Chen ${ }^{1,2,3}$, Shengchun Piao ${ }^{1,2,3,{ }^{*}}$, and Longhao Qiu ${ }^{1,2,3}$ \\ ${ }^{1}$ Acoustic Science and Technology Laboratory, Harbin Engineering University, 150001 Harbin, China \\ ${ }^{2}$ Key Laboratory of Marine Information Acquisition and Security (Harbin Engineering University), Ministry of Industry and \\ Information Technology, 150001 Harbin, China \\ ${ }^{3}$ College of Underwater Acoustic Engineering, Harbin Engineering University, 150001 Harbin, China
}

\begin{abstract}
In this work, a finite size acoustic vector sensor (AVS) array is designed and its performance is theoretically and experimentally studied. The two-dimensional AVS array is comprised of five vector sensors and configured as a cross, and the array carrier is a cylindrically symmetric structure. Theoretical analysis and simulation indicate that the proposed method considering structure scattering can widen the working bandwidth. Furthermore, the utilization of vector sensor enables a significant white noise gain improvement at low frequencies, which makes the array more robust and easier to realize. Experiments have been done to study the array performance from several aspects including sensor noise characteristics, the beampattern, the direction-of-arrival (DOA) estimation ability and the array gain. From the change of the sensor directivity patterns or the amplitude distortion of the noise field, we can clearly observe the scattering field intensity. Moreover, it shows that the influence of the structure scattering on the tangential component of the vector field is symmetric, while that of the radial component is asymmetric. Experimental results also demonstrate that, with the proposed method, the $2^{\text {nd }}$ and the $3^{\text {rd }}$ order beamformers can be obtained which could be further used for the estimation of target DOA. In addition, an array gain of at least $6 \mathrm{~dB}$ is obtained capable of detection of weak signals. Our results indicate that the proposed array with a physical size less than one meter, although affected by nearby scatterers, can effectively break the Rayleigh limit and realize the remote detection in lowfrequency regime.
\end{abstract}

\section{Introduction}

Compared to traditional half-wavelength arrays, finite size arrays whose sensors are compactly arranged have attracted a significant amount of interest due to their great potential to provide high angular discriminations and signal-to-noise (SNR) gains with relatively small sizes[1-3]. Distinguished the scale pressure sensor, a single acoustic vector sensor (AVS) can be regarded as the simplest finite size array[4], for it can provide a directivity improvement up to $6 \mathrm{~dB}$, and the directivity is not dependent on the frequency of sound. Although existing scientific literature on AVS arrays is overwhelmingly based on the traditional design of halfwavelength spacing, research on finite size AVS array starts to surge.

The finite size array technology looks quite attractive but is hard to implement in practice, especially in underwater applications. Some issues need to be settled such as the approximation (truncation) error in its beamforming theories, the sensitivity of the array, and the near-filed acoustic diffraction by the carrier or platform. Recently, $\mathrm{Ma}[5]$ proposed an analytical and closed-form optimal solution which solved the first problem. And a series of robust beamforming methods are proposed involving the sensitivity issue[6-7]. Compared to the beamformer sensitivity problem to errors, the acoustic field distortion problem caused by surrounding scatterers needs to be emphasized. There are some literatures considering the acoustic field when the shapes of scatterers are simple: Kosobrodov et al.[8] show an experimental comparison of the noise fields diffracted by the spherical equipment carrier or not, Meyer[9] discusses the beam-forming problem of a circular microphone array mounted on spherically shaped objects, while Zou and Nehorai[10] present a DOA estimation approach using miniature circular AVS array mounted on a cylinder. However, the size of finite size array is generally too small to be installed on equator of the carrier, or the array configuration demands it to be installed on one side of the platform. Additionally, the type of baffle varies from each other, and is commonly combined by different parts. All these add complexity to the theoretical model and its computation.

This paper tries to consider the above-mentioned limitations, and presents a more practical beamformer

Corresponding author: piaoshengchun@hrbeu.edu.cn 
for the finite size AVS array taking any cylindrically symmetric baffle into account, and the size of baffle is comparable to that of array, in which case the array and baffle comprise a self-contained system. Robustness of the beamformer is analysed, it shows that the structure scattering effect can widen the array's working bandwidth, and vector component of acoustic field makes it more robust to realize at low frequencies. Experimental data shows the efficiency of the method. This paper is organized as follows. Section II describes the array configuration and signal models for pressure and vector sensor. The beamformer design method is outlined in Section III, as well as the simulations of array performance. And then, experimental test and discussions such as the noise characteristics, the beampattern, the DOA estimation ability and the array gain are separately demonstrated in Section IV. And conclusions are drawn in Section V.

\section{Array configuration and signal model}

Finite size array needs to be installed on the platform or an equipment carrier. Thus, the carrier (platform) will react to the incident wave and distorts the surrounding acoustic filed. We will consider the scattering of these nearby objects and the corresponding array beamforming method as follows. The carrier consists of a thin plate and an elastic spherical shell, the plate is designed to mount the array sensors, and the thin spherical shell with outer radius $a$ is used to enclose the electronic equipment. The array and spherical shell are installed on opposite side of the plate, as seen in Fig.1. Such an arrangement of the equipment is widely used in practice. The region of interest is the horizontal plane where the twodimensional finite size AVS array stays.

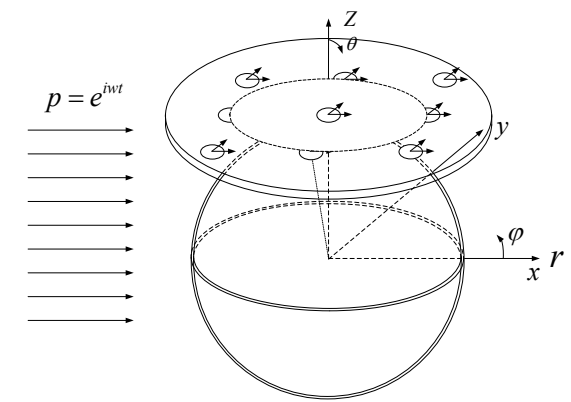

Fig. 1. Schematic illustration of the system

When solving the spherical shell scattering problem, we can use the analytical method to solve the scalar Helmholtz equation. And the resulting pressure and vector field can be expressed as

$$
\begin{gathered}
p\left(\vec{r}, \overrightarrow{k_{s}}\right)=\sum_{m=-\infty}^{\infty} e^{i m\left(\varphi-\varphi_{s}\right)} C_{m} \\
{\left[\begin{array}{l}
u_{x}(\vec{r}) \\
u_{y}(\vec{r})
\end{array}\right]=\boldsymbol{T}(\vec{r})\left[\begin{array}{l}
u_{r}(\vec{r}) \\
u_{\theta}(\vec{r}) \\
u_{\varphi}(\vec{r})
\end{array}\right]=\left[\begin{array}{l}
\sum_{m=-\infty}^{\infty} C_{m_{-} x} e^{i m\left(\varphi-\varphi_{s}\right)} \\
\sum_{m=-\infty}^{\infty} C_{m_{-} y} e^{i m\left(\varphi-\varphi_{s}\right)}
\end{array}\right]}
\end{gathered}
$$

where

$$
C_{m}=\sum_{l=|m|}^{\infty} B_{l}\left(k_{2} r\right) \frac{(l-|m|) !}{(l+|m|) !} P_{l}^{|m|}\left(\cos \theta_{s}\right) P_{l}^{|m|}(\cos \theta)
$$

$$
\begin{gathered}
B_{l}(k r)=i^{l}(2 l+1)\left[j_{l}(k r)+A_{l} h_{l}(k r)\right] \\
{\left[\begin{array}{l}
C_{m_{-} x} \\
C_{m_{-} y}
\end{array}\right]=\boldsymbol{T}(\vec{r})\left[\begin{array}{l}
C_{m_{-} r} \\
C_{m_{-} \theta} \\
C_{m_{-} \varphi}
\end{array}\right]}
\end{gathered}
$$

and $\boldsymbol{T}(\vec{r})$ is the transfer matrix between the Cartesian coordinate system and spherical coordinate system. It is obvious that the partial velocity field along $x$ - and $y$ direction can also be expressed in the Fourier series expansion form.

The Kirchhoff integral is used to generate the thin plate scattering field solution. According to the Maggi transfer, at the low-frequency and small grazing angle condition, the total field around the thin plate can be approximately expressed as

$$
\Phi(P)=\Theta \Phi_{i}(P)+\frac{e^{i k x_{0}}}{x_{0}} \frac{a^{2} e^{i k R}}{R^{2}\left(1+\frac{h}{R} \sin \theta_{0}\right)} \cdot\left[\begin{array}{l}
\sin \theta_{0}\left[J_{0}\left(\frac{k a x}{R}\right)+J_{0}\left(\frac{k a y}{R}\right)\right] \\
+\frac{\left(x \sin \theta_{0}+h \cos \theta_{0}\right)}{a} i J_{1}\left(\frac{k a x}{R}\right) \\
+\frac{y}{a} \sin \theta_{0} i J_{1}\left(\frac{k a y}{R}\right)
\end{array}\right]
$$

Considering the properties of Bessel functions, the second and third terms in brackets can be neglected because of their small quantities, and the zero-order Bessel function thus plays a major role. With further analysis, we can conclude that: it is appropriate to consider the scattering filed remains unchanged within the interested conditions; due to the small size of the plate, the scattering field will not undulate, but is monotonic with the distance; what's more, the scattering field is symmetric about the incident wave azimuth.

For situation where the spherical shell and the thin plate coexist, the surrounding acoustic field and its property can be studied by the Finite Element Method. Conclusion can be drawn which is consistent with the thin plate situation. It is reasonably to infer that as long as the carrier is cylindrically symmetric, the recorded acoustic field will have the form of Eq.(2), only that the coefficient items $C_{m}$ will be changed referring to the material and shape of the baffle.

Thus, for the problems mentioned in this paper, we can write the manifold of the AVS array as follows:

$$
\begin{aligned}
\mathbf{v}\left(\theta_{s}\right) & =\left[v_{1}\left(\varphi_{s}\right), \ldots, v_{N}\left(\varphi_{s}\right)\right]^{T}, n=1,2, \ldots, N \\
v_{n}\left(\varphi_{s}\right) & =\sum_{m=-\infty}^{\infty} e^{i m\left(\varphi_{n}-\varphi_{s}\right)} C_{m_{-} n}=\sum_{m=-\infty}^{\infty} e^{-i m \varphi_{s}} D_{m_{-} n} \\
D_{m_{-} n} & =e^{i m \varphi_{n}} C_{m_{-} n}
\end{aligned}
$$

Theoretically, the coefficients $C_{m_{-} n}$ in Eq.(5b) are known. However, for situations where the carrier structure consists of many parts that piecing together or there are carrier material losses, the coefficients $C_{m_{-} n}$ cannot be precisely calculated. Fortunately, the finite size AVS array systems are small enough to be practically tested or measured, thus the actual value of $C_{m_{-} n}$ can be achieved conveniently.

\section{Beamformer design and simulations}




\subsection{Beamforming algorithm}

Several performance metrics are chosen such as directivity, front-back (port-starboard) ratio, and main beam width when design array beamformers. An alternative way is to choose the beamformer weighting factors such that the beamformer output corresponds to a certain desired response function. The latter approach is pursued here with a desired beampattern in the form of Eq.(6)

$$
B_{M P}\left(\vartheta, \varphi_{s}\right)=\sum_{m=0}^{M} a_{m} \cos \left[m\left(\vartheta-\varphi_{s}\right)\right]
$$

where $\vartheta$ is the steering angle. The beamformer output maximizes when $\vartheta$ equals $\varphi_{s} . M$ is called the order of the beampattern, $a_{m}$ are real coefficients and different values of these coefficients determine the different directivity pattern.

To apparently correspond to the signal expansions in Eq.(5), we rewrite Eq.(6) in another fundamental way

$$
\begin{aligned}
B_{M P}\left(\vartheta, \varphi_{s}\right) & =\frac{1}{2} \sum_{m=0}^{M} a_{m}\left[e^{i m\left(\vartheta-\varphi_{s}\right)}+e^{-i m\left(\vartheta-\varphi_{s}\right)}\right] \\
& =\sum_{m=-M}^{M} b_{m} e^{i m\left(\vartheta-\varphi_{s}\right)}=[\mathbf{b} \gamma(\vartheta)]^{T} \mathbf{e}\left(\varphi_{s}\right)
\end{aligned}
$$

where

$$
\begin{aligned}
& \mathbf{b}=b_{m}=b_{-m}=\left\{\begin{array}{cc}
a_{m} & m=0 \\
a_{m} / 2 & m \neq 0
\end{array}\right. \\
& {\left[b_{-M}, \cdots, b_{0}, \cdots, b_{M}\right]^{T}} \\
& \gamma(\vartheta)=\operatorname{diag}\left[e^{-i M \vartheta}, \cdots, 1, e^{i M \vartheta}\right] \\
& \mathbf{e}(\phi)=\left[e^{i M \phi}, \cdots, 1, \cdots, e^{-i M \phi}\right]^{T}
\end{aligned}
$$

The superscript $T$ is the transpose operator. The beamformer is designed that, when a set of complex weights are applied to the output of array sensors, the weighted outputs are then summed to form the fixed beamformer output

$$
\begin{aligned}
B(\mathbf{W}(\omega, \vartheta)) & =\sum_{n=1}^{N} w_{n}(\omega, \vartheta) \sum_{m=-M}^{M} D_{m_{-} n} e^{-i m \varphi_{s}} \\
& =\sum_{m=-M}^{M} e^{-i m \varphi_{s}} \sum_{n=1}^{N} D_{m_{-} n} w_{n}(\omega, \vartheta)
\end{aligned}
$$

where

$$
\mathbf{W}(\omega, \vartheta)=\left[w_{1}(\omega, \vartheta) w_{2}(\omega, \vartheta) \cdots w_{N}(\omega, \vartheta)\right]^{T}
$$

Now, let $B_{M P}\left(\vartheta, \varphi_{s}\right)$ in Eq.(7) to be same as $B(\mathbf{W}(\omega, \vartheta))$ in Eq.(9), we have

$$
\mathbf{W}(\omega, \vartheta)=\Psi^{H}\left(\boldsymbol{\Psi} \Psi^{H}\right)^{-1}[\mathbf{b} \gamma(\vartheta)]
$$

where

$$
\begin{aligned}
& \boldsymbol{\Psi}=\left[\boldsymbol{\psi}_{-M}, \cdots, \boldsymbol{\psi}_{0}, \cdots \boldsymbol{\psi}_{M}\right]^{T} \\
& \boldsymbol{\psi}_{m}=\left[D_{m_{-} 1}, \cdots, D_{m_{-} 2}, \cdots, D_{m_{-} N}\right]
\end{aligned}
$$

And the white noise gain $(W N G)$, which is an efficient measure to evaluate the sensitivity of the array beamformer to its imperfections as well as other uncertainties, can be easily achieved as

$$
W N G=10 \log _{10} \frac{1}{[\mathbf{b} \gamma(\vartheta)]^{H}\left(\Psi \Psi^{H}\right)^{-1}[\mathbf{b} \gamma(\vartheta)]}
$$

It can be seen that when carrier exists, the coefficient $D_{m_{-} n}$ contains not only the eigenfunctions associated with the incident component, but also that with the scattering component. The zeros of these different types of eigenfunctions occurr at different frequencies, thus, their combination generally does not have zeros. Consequently, the performance degradation caused by nulls can be significantly improved, and the working frequency band will be broadened.

\subsection{Simulations}

Several factors are discussed to testify their impact on the $W N G$ performance, they are the baffle scattering, the utilization of vector sensor and the sensor number.

Fig. 2 give some WNG comparisons of the baffle with different boundary conditions, the spherical baffle is considered. The simulation conditions are: the radius of AVS array is $0.4 \mathrm{~m}$; the radius of the baffle is $0.3 \mathrm{~m}$; the center of the spherical baffle is set $0.35 \mathrm{~m}$ lower than the horizontal plane of the AVS array; the different boundary conditions are transparent, rigid and soft; sensor number is set to be 5 or 12 .

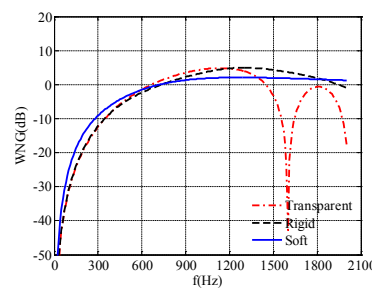

(a) $N=5$

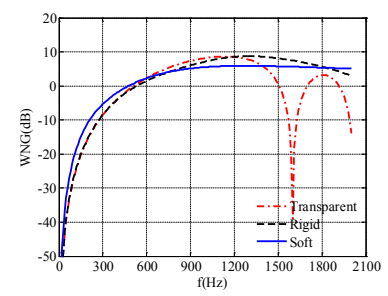

(b) $N=12$
Fig. 2. WNG for different boundary conditions.

Compared to the acoustic transparent cases, the rigid and soft baffle cases can both have almost frequencyinvariant $W N G \mathrm{~s}$, which are consistent with the theoretical analysis. The $W N G$ improvement with sensor number can also find a good agreement with Eq.(13). Notice that below $600 \mathrm{~Hz}$, the ratio of wavelength to the size of baffle is relatively large, and the incident wave can passby the baffle, thus the pressure field is nearly unchanged for rigid object whose wave impendence is sufficiently large. While for soft baffle, the pressure is approximate to zero and the phase of diffraction wave is opposite to that of the incident wave on the boundary. In this case, the pressure field is severely affected. For most underwater cases, the baffle is elastic, thus the $W N G$ is between that of soft and rigid.

In the following figure, simulation results will be shown for finite size acoustic pressure and vector sensor arrays with radius of $0.4 \mathrm{~m}$ 


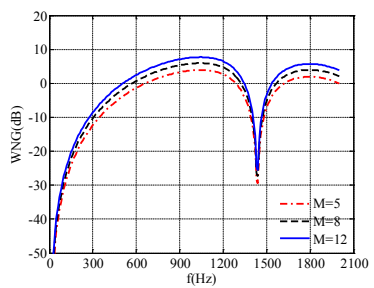

(a) pressure sensor array

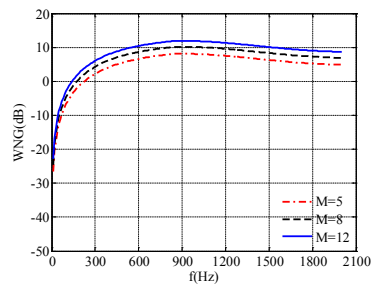

(b) finite size AVS array
Fig. 3. WNG for different arrays.

In Fig.3(b), the null has been midigated, and $W N G$ improves with the sensor number increases. What's more, the AVS has a significant $W N G$ improvement at the low frequency regime. Within range of $200-600 \mathrm{~Hz}$, the $W N G$ of finite size AVS array is no less than $0 \mathrm{~dB}$, which provides a foundation to the realization of finite size AVS array in practice.

\section{Experimental results and discussions}

A prototype of a two-dimensional finite size AVS sonar array (see Fig.4) has been developed to validate the proposed algorithm. The array consists of five vector sensors with inter-sensor spacing $d=0.4 \mathrm{~m}$. The sensor located at the centre of the array is numbered 5, and the others are numbered from 0 to 4 in the anticlockwise direction. An elastic cylindrical shell has been designed as the carrier. Thus, the sound field distorts due to the diffraction of the carrier. All the sensors have been tested and selected primarily to ensure their normal work.

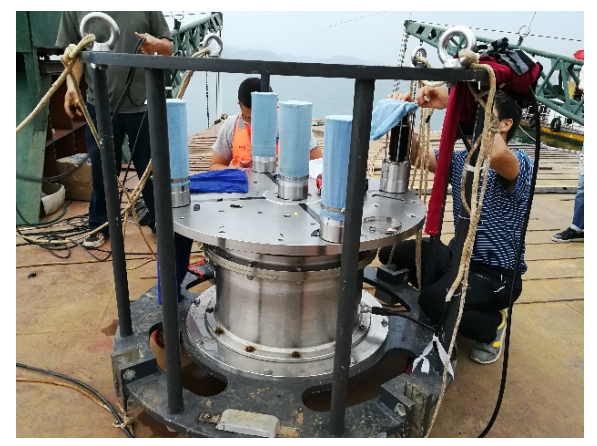

Fig. 4. Experimental finite size AVS array.

Array performance such as the noise characteristics, the beampattern, the DOA estimation ability and the array gain will be discussed.

\subsection{Noise characteristics}

The periodogram of noise received by vector sensors are displayed in Fig.5. The upper and lower periodogram in each subfigure represent the $x$-component and $y$ component of the recorded acoustic field, separately.
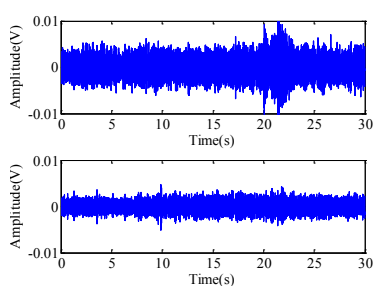

(a) sensor $1 \#$
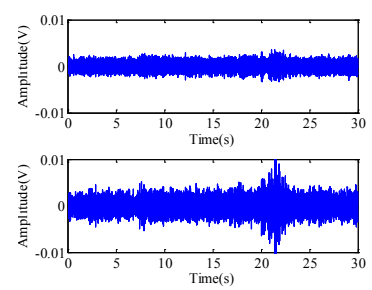

(b) sensor $2 \#$

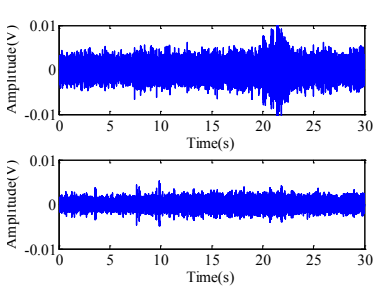

(c) sensor $3 \#$
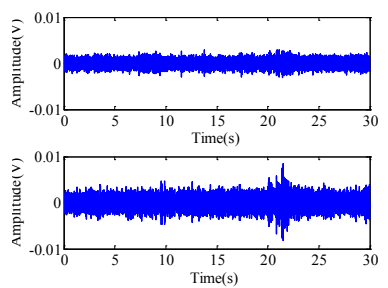

(d) sensor 4\#

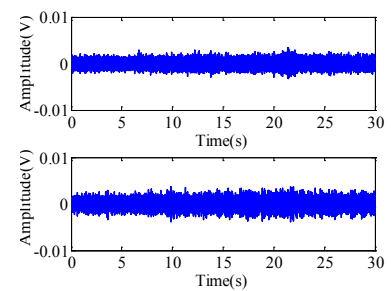

(e) sensor $5 \#$

Fig. 5. Periodograms of noise received by vector sensors.

It is obvious that the amplitudes of radial directional components are higher than the tangential components. This testifies the scattering effect of the carrier. With further study, it is possible to tell the scattering intensity through this phenomenon.

\subsection{Beampattern}

Considering the symmetry of the array configuration, the directivity patterns of sensor $3 \#$ and $4 \#$ will be similar to that of $1 \#$ and $2 \#$, respectively. Due to space limitations, we just display the amplitude responses of sensor $5 \#, 1 \#$ and 2\# here in Fig.6.
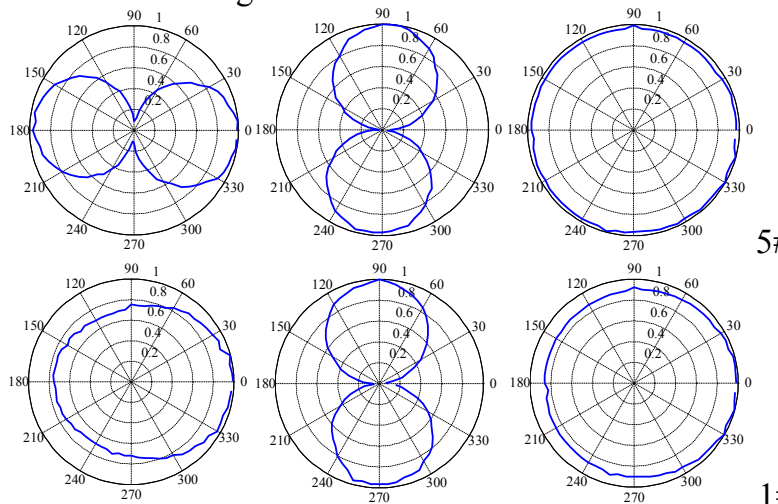

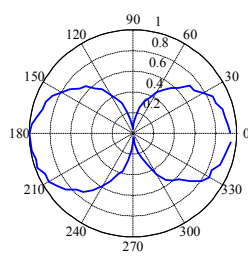

$x$

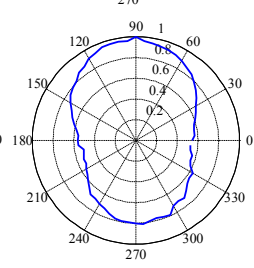

$y$

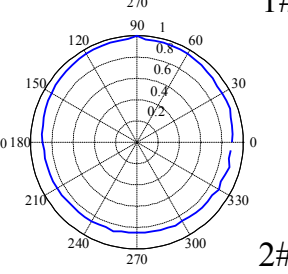

$p$
Fig. 6. Practical amplitude responses of sensor 5\#, 1\# and $2 \#$.

Theoretically, the directivity of acoustic pressure field is omnidirectional and that of the vector field has dipole pattern, which have found good agreements in sensor 5. This situation stands for the $y$-component of sensor 1 and the $x$-component of sensor 2, who are the tangential components of the acoustic vector field. While for the $x$-component of sensor 1 and $y$-component of sensor 2, who are the radial components of the acoustic vector field, large distortions have been observed, and 
the nulls of the directivity pattern disappear. It's reasonable to suspect that the distortion is caused by scattering of the carrier.

The practical $2^{\text {nd }}$ and $3^{\text {rd }}$ order beams with steering angle $90^{\circ}$ are shown in Fig.7. The curves with squared markers illustrate the proposed beampatterns with our method, and the curves with triangle markers show the beam-forming result without considering the scattering effect. The theoretical beam patterns (no markers) are also illustrated for comparison.

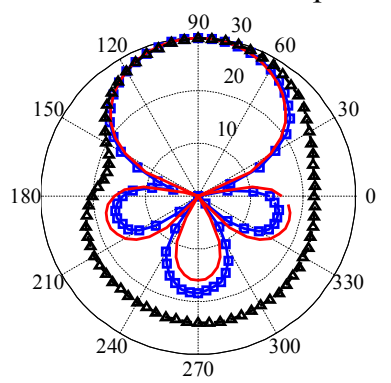

(a) $2^{\text {nd }}$ order

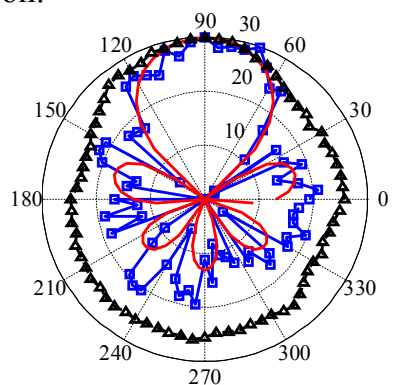

(b) $3^{\text {rd }}$ order
Fig. 7. The practically measured beampatterns.

It can be observed that the practical $2^{\text {nd }}$ order beampattern using the proposed method is in good agreement with the theoretical beampattern, and the nulls occur at the right place. The $3^{\text {rd }}$ order measured beampattern shows some distortions while the main lobe still exists and the side lobes diverge from the theoretical one. These phenomena accord with the discipline that sensitivity increases as order $M$ goes up. Compared to the proposed beamformer, the beampatterns taking no account of the baffle have no obvious main lobes, thus failed to give the right outputs.

\subsection{DOA estimation ability}

Fig. 8 and Fig.9 display the spatial spectral and track of the target separately. In Fig.9, the triangle markers illustrate the estimated DOA results while circles show the actual ones achieved from the GPS.

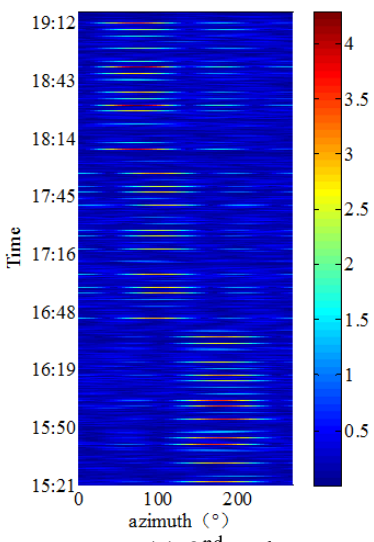

(a) $2^{\text {nd }}$ order

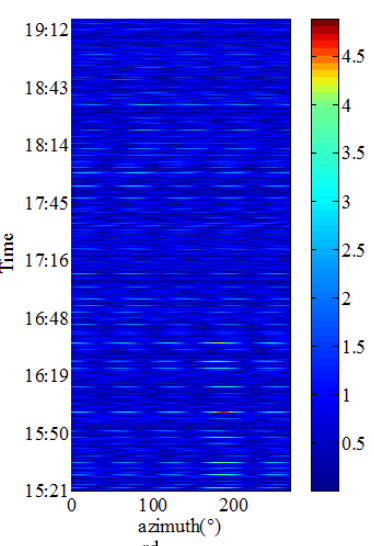

(b) $3^{\text {rd }}$ order
Fig. 8. Spatial spectral.

As can be seen, spatial spectral obtained from the $2^{\text {nd }}$ order beamformer have wider main lobes while those from $3^{\text {rd }}$ have higher side lobes and worse robustness, and the DOA estimation results are basically same to those of the actual ones. Among the three transmitting intervals (incident angle are $180^{\circ}, 118^{\circ}$ and $73^{\circ}$, respectively), the results for the first and third intervals which have more coaxial incident waves are very precise, while the second interval has a mean degree of 17.5 diverge from the actual one. The variances for these intervals are $3^{\circ}, 3.5^{\circ}$ and $2.1^{\circ}$, respectively.

As for the $3^{\text {rd }}$ situation, the target DOA estimation succeeds in the first interval with a variance of $1.4^{\circ}$, which is lower than that of $2^{\text {nd }}$ order case. Conclusion can be drawn that the proposed finite size AVS array can realize robust $2^{\text {nd }}$ and $3^{\text {rd }}$ order beamformer when carrier scattering exists, and they can be used to further applications, only that the $3^{\text {rd }}$ order depends.

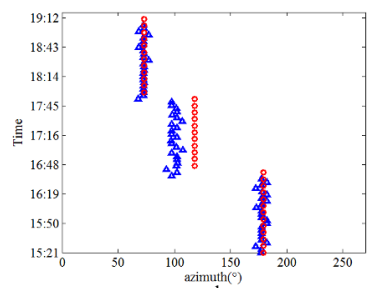

(a) $2^{\text {nd }}$ order

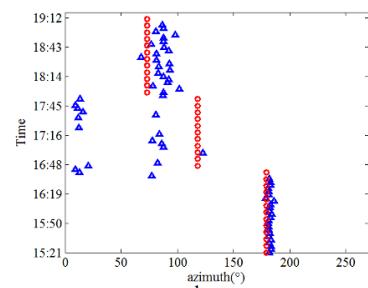

(b) $3^{\text {rd }}$ order
Fig. 9. DOA results.

\subsection{Array gain}

The array gain will be contrastively illustrated in Fig.10 and Fig.11, Fig. 10 gives the LOFAR results while Fig.11 displays the periodogram results of narrow-band filtering.

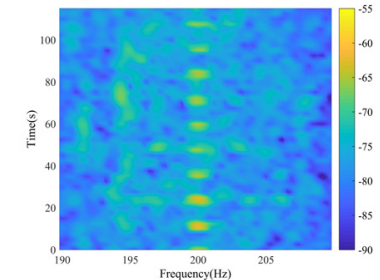

(a) Pressure channel of sensor 5\#

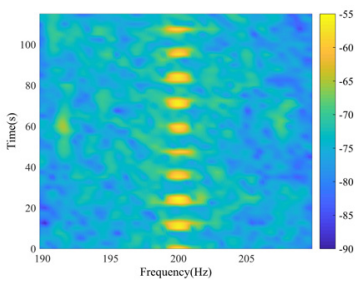

(b) Array output
Fig. 10. Comparison of LOFAR results.
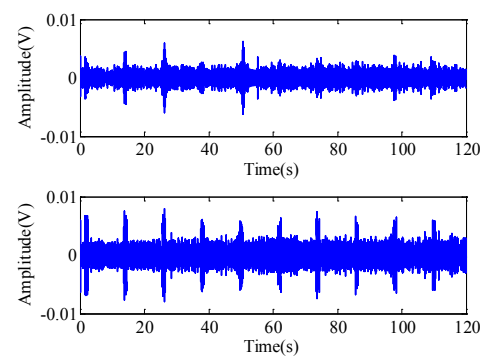

Fig. 11. Comparison of periodogram results.

Both results have testified the validation of the finite size vector sensor array and the proposed method. And array gain of at least $6 \mathrm{~dB}$ is achieved, which is capable of detecting weak signals.

\section{Conclusions}

This paper studies the design of finite size AVS array beamformer taking the surrounding carrier scattering into account. We focus on the self-contained system comprised by the array and the cylindrically symmetric carrier. The $W N G$ characteristic for sensor array affected by baffle scattering or for AVS array is theoretically 
analysed and numerically simulated. It has been found that the working bandwidth can be widened significantly; meanwhile, the AVS array can provide a great $W N G$ improvement at low frequencies. Experiments have been carried out to validate the proposed method. Amplitude distortion of the noise field or the sensor directivity patterns are illustrated, from which we can clearly observe the scattering field intensity. Results also indicate that the $2^{\text {nd }}$ and $3^{\text {rd }}$ order beampatterns are achieved, and the array can be successively used in target DOA estimation. Array gain of at least $6 \mathrm{~dB}$ is obtained, which indicate that our approach can provide a practical solution to finite size AVS array in underwater acoustic remote detection at low frequency regime.

\section{References}

1. J. Benesty, J. Chen, Study and design of differential microphone arrays (Berlin: Spinger, 2012)

2. B. Rafaely, J. Acoust. Soc. Am. 116(4), 2149-2157 (2004)

3. G. W. Elko, J. Acoust. Soc. Am. 105(4), 1098 (1999)

4. B. A. Cray, A. H. Nuttall, J. Acoust. Soc. Am. 110(1), 324-331 (2001)

5. Y. Ma, Y. Yang, Z. He, K. Yang, et.al, IEEE Trans. On. Industrial Electronics. 60(1), 203-209 (2013)

6. L. Zhao, J. Benesty, and J. Chen, IEEE Trans. On Audio, Speech, and Language Processing. 22(10), 1455-1466 (2014)

7. G. Huang, J. Benesty, J. Chen. J. Acoust. Soc. Am. 141(5), 3236-3249 (2017)

8. R. A. Kosobrodov, V. N. Nekrasov, Acoust. Phys. 47(3), 323-328 (2001)

9. J. Meyer. J. Acoust. Soc. Am. 109(1), 185-193 (2001)

10. N. Zou, A. Nehorai. IEEE Trans. Signal Processing. 57(8), 3041-3052 (2009) 